\title{
Autofocus in infrared microscopy
}

\author{
Raphael Abele* ${ }^{\dagger}$, Daniele Fronte*, Pierre-Yvan Liardet*, Jean-Marc Boi ${ }^{\dagger}$, Jean-Luc Damoiseaux ${ }^{\dagger}$, Djamal Merad ${ }^{\dagger}$ \\ * STMicroelectronics \\ Rousset, France \\ raphael.abele, daniele.fronte, pierre-yvan.liardet @ st.com \\ ${ }^{\dagger}$ Laboratoire d'Informatique et Systèmes \\ Aix-Marseille University \\ Marseille, France \\ raphael.abele, jean-marc.boi, \\ jean-luc.damoiseaux, djamal.merad @lis-lab.fr
}

\begin{abstract}
Autofocus (AF) is a widely investigated subject in the fields of natural scene images, industrial assembly and biologic microscopy. This paper proposes a new effective AF method for infrared (IR) microscopy in the context of the Integrated Circuit industry (IC). The proposed method operates in the wavelet domain using a custom orthogonal wavelet for the 2D Discrete Wavelet Transform (DWT). The quality criterion of our AF algorithm relies on the standard deviance of the DWT coefficients, computed per subband and per level. Tested on several optical magnifying lenses, our method is robust time-efficient, and usable on-the-fly in the IC location system.

Index Terms-Infrared imaging, Microscopy, Focusing, Image analysis, Image quality, Image decomposition, Wavelet transforms, Statistics
\end{abstract}

\section{INTRODUCTION}

Integrated Circuits $\left(\mathrm{ICs}^{1}\right)$ are electronic components whose applications seem unlimited: they are used in many fields such as wearable technologies and IoT (Internet of objects). In order to protect the privacy of users, some ICs need to be secured, and their security needs to be validated. One way to characterize a secure IC is to study its behavior following a physical disruption. Such disruption may be obtained by a laser shot targeted on the internal structures of the IC. The accuracy of the shot relies on the precision of the laser power calibration and 3D positioning inside the IC. In our study, the Object Of Interest (OOI) is considered as made of three layers of different materials: (1) the silicon, (2) a conductor material forming the conductive tracks, and (3) an electrical insulator, as illustrated in Fig. 1.

The internal OOI structures are visible on the surface of the conductive tracks, which is the Surface Of Interest (SOI). The

Project funded by the Association Nationale de la Recherche et de la Technologie (ANRT).

${ }^{1} \mathrm{~A}$ glossary is present at the end of this paper.

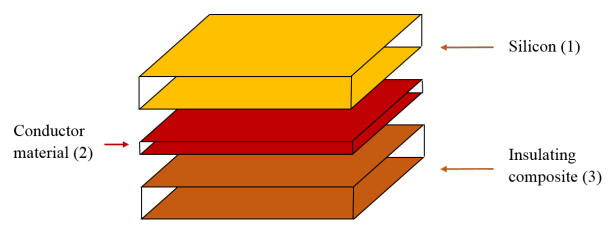

Fig. 1: Concept mapping of three materials composing our IC.
3D positioning of the laser on the SOI is done with an infrared (IR) camera that can see through the silicon layer. In our work, the optical system and the laser source are interdependent, so the optical adjustment implies laser position adjustment. Usually, these adjustments are made manually by skilled men, which raises two problems: time loss and non-reproducibility of the characterization (because of potential imprecision). An autofocus (AF) system could partially solve this problem by automating one dimension of the $3 \mathrm{D}$ positioning process.

Section (II) shortly introduces some works related to the AF. Then, section III presents our particular work environment. Section IV describes the AF method we propose, and section $\mathrm{V}$ details the overall experiment results.

\section{AROUnd THE AUTOFOCUS}

The AF mechanism is a deterministic algorithm that determines the lens position for which the system is "well focused" on the scene/object of interest (see Fig. 2), that is, the position for which the image of the scene or object is sharpest.

Two AF approaches exist: active and passive. The active approach depends on an additional system measuring the distance from the system to the scene/object. For example, ultrasonic sound waves and IR light reflection are two ways of measuring distances. Once the optics-scene distance is known, the correct lens position to obtain a focused image is estimated. Such an active system is not available, so we consider a passive approach relying on image analysis. This analysis determines the most relevant object/scene image, which we then use to find the correct focus. At this point, we are confronted with the issue of Image Quality Assessment (IQA).

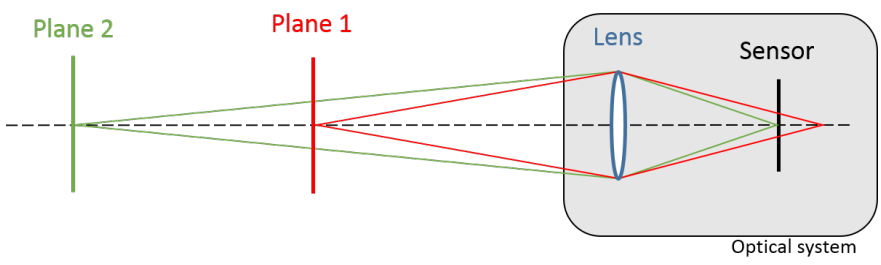

Fig. 2: Minimalist optical system; the image on plane 2 (green) is well focused on the optical sensor whereas the image on plane 1 is not. 
The Human Visual System (HVS) is the most reliable tool for IQA: the main difficulty is the interpretation of human subjectivity with algorithms following objective rules and criteria [1].

Some subjective methods attempt to emulate HVS using the Mean Opinion Score, but they are time-consuming and generally non-deterministic; these methods are partly based on objective metrics pooled together to predict the quality score [2].

Objective IQA methods attempt to be correlated with the HVS. Depending on the amount of available information from the image source, methods are categorized as Full-Reference (FR) based if an original, non-distorted image is available [3] or No-Reference (NR) based otherwise [4]. If just some of the original image features are known, the method is qualified as Reduced-Reference (RR) based. See reference [5] as an example. RR-IQA methods are generally guided/optimized NR-IQA methods. In the AF context, both of the NR and RR-IQA methods are used, since the quality criterion is hypothetical. Images are ranked according to this criterion, and the best-ranked image is associated with the correct lens position.

Statistics of order 1, 2 and higher are widely used in signal processing since the 1960's. In image analysis, each statistic point out an image feature [6]:

- Average Gradient (AG): reflects the contrast and the clarity of the image. It can be used to measure the spatial resolution of a test image, where a larger average gradient indicates better spatial resolution.

- Mean metric: indicates the image average brightness level. For equivalent scenery, image brightness increases with the mean.

- Auto-Correlation (AC): spotlight redundant data. If an image is blurred or the edges are smoothed, the correlation between neighboring pixels becomes high.

- Entropy metric: measures the information quantity of an image. If the probability of occurrence of each gray level is low, the entropy is high, and vice versa.

- Standard Deviation (SD): reflects the contrast of the image; the image contrast increases with the SD.

- Kurtosis metric: a statistical measure of the degree of sharpness or flatness of a distribution (i.e., average slope and energy concentration). Increases with the depth of focus.

- Skewness metric: a statistical measure of the direction and extent to which a data set deviates from a distribution. For a standard normal distribution, high skewness indicates asymmetry of the data. In this case, the data contains a greater amount of information.

These statistics can be computed on the image data or on its transform, that is, in spatial, frequency or time-frequency domains.

Since AF algorithms rely on specific image analysis, they cannot be generalized. Several methods exist, each adapted to its context such as images of natural scenes [5], [7]-[9], low-contrast images [10] or microscopic images [11]-[13]. In paper [13], the author studied several autofocus methods in a particular case (lipid droplets inside microscopic worms). The focus criterion is clarity-evaluation based. He tested the performance and accuracy of sixteen autofocus methods: histogram, intensity, statistic, derivative and transform-based. He found that the absolute Tenengrad algorithm had the best performance against accuracy for its specifics. Finally, each approach uses well justified metrics. In our proposal we define metrics adapted to IR microscopy for the IC industry, which implies some constraints (see Section III).

\section{WORK ENVIRONMENT}

\section{A. Available material}

Observation of the SOI under the silicon layer is possible thanks to its optical properties. Indeed, around the wavelength $1 \mu \mathrm{m}$ and higher, the silicon absorption coefficient is not significant [14], [15]. In other words, silicon is "transparent" to lights whose wavelengths are around $1 \mu \mathrm{m}$ and above (such as IR light).

Our motorized optical system provided by $\mathrm{AlphaNov}^{2}$ is composed of:

- an uncooled IR camera

- an optical microscope with 4 magnifying lenses $(2.5 \mathrm{x}$, $5 x, 20 x$ and 50x) allowing us to observe the OOI (around $5 \mathrm{~mm}^{2}$ ) and its internal structures (micro-metric scale)

Three types of IR camera are available on the market: LongWave, Mid-Wave and Short-Wave (LWIR, MWIR and SWIR, respectively). On the one hand, MWIR and LWIR sensors detect thermal emissions from objects, and are efficient when these are warmer than their surroundings. On the other hand, SWIR cameras use the reflected light, much like the slightly shorter wavelengths of the visible spectrum (see Fig. 3). In our experiment we worked with the latter type of camera.

With the unique capabilities of our SWIR camera, we are able to see the SOI through the silicon layer. To propose an automated method for focusing on the SOI, our algorithm must take in account some specifics arising from the camera and from the SOI (see Section III-B).

\section{B. Constraints and special features}

\section{Concerning the camera:}

${ }^{2}$ www.alphanov.com

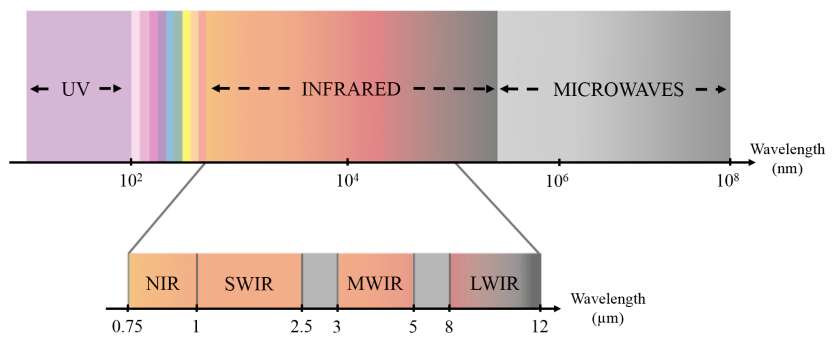

Fig. 3: Infrared wavelength in the electromagnetic spectrum. 
The IR camera sensor is an InGaAs, sensitive to a large IR wavelength range $(900 \mathrm{~nm}$ to $1700 \mathrm{~nm})$; the embedded Wide Dynamic Range technology (WDR) dynamically detects the relevant wavelengths to take into account for best image quality. Despite its accuracy, this camera stays sensitive to external thermal interference. Since the sensor is uncooled (thermally not insulated), the thermal instability of the environment produces thermal noise, which affects the image acquisition (see Fig. 4).

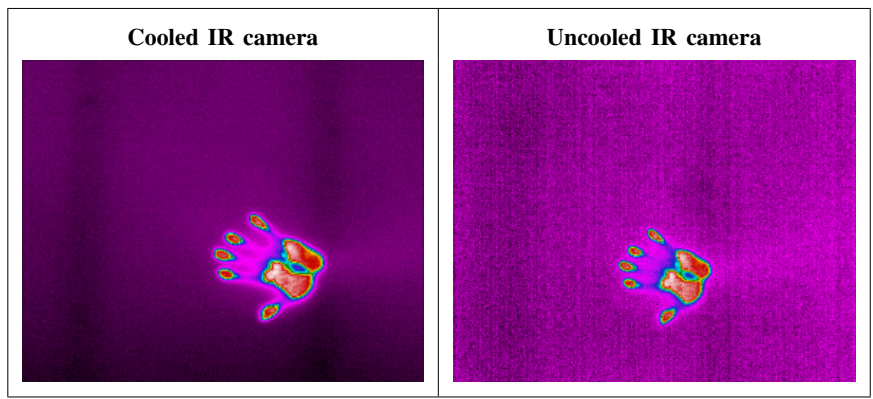

Fig. 4: An example of images acquired with cooled and uncooled infrared cameras (source: flir.com).

\section{Concerning the object of interest:}

Two distinct surfaces are considered for the autofocus algorithm: the silicon and the SOI. As said in III-A, silicon is transparent to our IR light and should not be visible. However, in many cases, contaminants (e.g. dusts) are ingrained on the silicon, which reveals the surface to any light. This implies a possible conflict of interest, considering the sharpness criterion: the silicon surface with its contaminants may be sharper than the SOI. This point underlines that sharpness is not, in practice, the appropriate criterion to exclusively characterize an SOI. Fig. 5 shows to the notable states during the autofocus process.

We may compare the conductive layer of an IC to a city, and its internal structures to buildings. As shown in
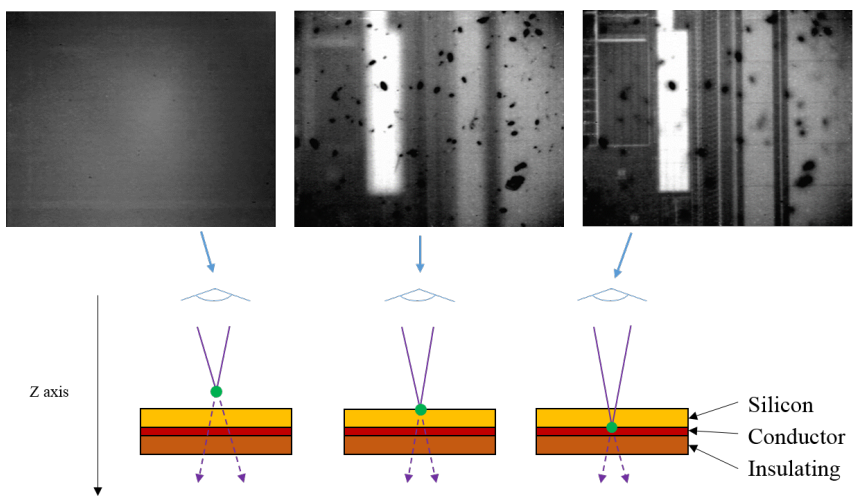

Fig. 5: Images acquired at several focal point positions (green points) according to the Z-axis. Component out of focus (left), focused on the silicon surface (middle) and on the conductive track surface (right).

Fig. 6, everything is orthogonally disposed, in regard to the rectangular shape of the component. This criterion is a good candidate for our AF algorithm.

However, a difficulty arises from the SOI image texture. An IC is built by microscopic material deposition, which implies a textural grain visible with a high magnifying lens $(20 \mathrm{x}$ and $50 x)$. Considering the SOI images, this grain can be compared to a noise in the images: structures are clusters of points, more or less dense (illustrated in Fig. 7). In the worst case, detection of the structure boundaries turns out to be difficult and the images could need preprocessing to be well treated.

To sum up, we may state that:

a. Sharpness is not a good criterion to make the difference between the silicon surface and the SOI.

b. On the SOI, horizontal and vertical structures boundaries are visible.

c. Because of textural grain and noise, topological information is not pertinent (e.g. line detection).

d. The AF method has to be effective for each magnifying lens $(2.5 \mathrm{x}, 5 \mathrm{x}, 20 \mathrm{x}$ and $50 \mathrm{x})$.

Since we know which features are expected in the targeted image (b.), our IQA can be qualified as RR-based. To avoid preprocessing such as image denoising (c.), we propose a transform-based analysis in the next section.

\section{AUTOFOCUS METHOD PROPOSAL}

The criterion analyzed for an AF system has to be strong enough to prevent false results. In that sense, for a robust AF algorithm, we need a robust quality metric.

\section{A. Analysis domain proposal}

As previously described, image preprocessing should be performed before any image-based analysis (e.g. denoising). But it could break information continuity in our images (e.g. spatial information), and could be time-consuming. To preserve this continuity, our approach is transform-based, which may also save computation time. This transform is described below.

\section{Wavelet transform:}

Several image transforms exists. One of the most commonly used is wavelet decomposition, but has limitation due to its orthogonality (i.e. there is little orientation information). Others exist such as the steerable pyramid and curvelet transforms:

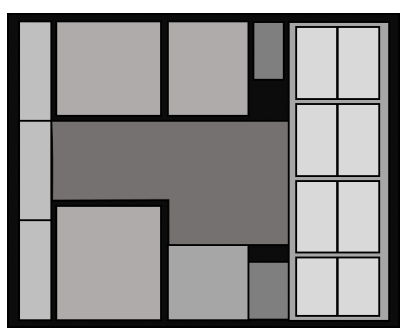

Fig. 6: A simple representation of an IC and its internal structures.
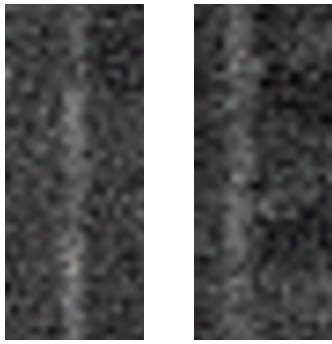

Fig. 7: Two lines delimiting structures boundary $(50 \mathrm{x})$. 
the steerable pyramid transform overcome this orientation limitation and the curvelet transform is an extension of the wavelet concept, with less redundancy and more orientation information [16]-[18]. However, according to the features described in section III-B, the main information needed for our analysis is the horizontal and vertical components of images. Therefore, we only consider wavelet decomposition for our IQA. Moreover, it may permit skipping some useless information related to material texture or even noise. For this purpose, the wavelet choice is important, considering that each wavelet matches specific signal information.

\section{Wavelet choice:}

Wavelets are used to decompose signal information, depending on their characteristics and topology. Here we need to find the best wavelet matching to the interesting information in our IR images, that is, the structures contours. Vu and Chandler [19] described a method to construct an image sharpness map based on 2D wavelet decomposition. The implementation of such a sharpness map could allow us to visually evaluate how image information is decomposed by a wavelet.

To that end, we took three photos of the SOI with $5 \mathrm{x}$, 20x and 50x magnifying lenses. For each image, we built a sharpness map considering the following: two orthogonal and two bi-orthogonal wavelets, respectively:

1. Haar wavelet [20], since the image can be interpreted as binary: reflexive versus non-reflexive materials, structures versus background (I).

2. A Custom Orthogonal Wavelet (COW) built from a father wavelet whose scaling coefficients are defined in (1) and and from which filter bank coefficients are computed in Tab. III. See its illustration in Tab. II.

$$
\phi=\left[\begin{array}{llll}
\frac{1}{k} & \frac{1}{k} & \frac{1}{k} & \frac{1}{k}
\end{array}\right]
$$

TABLE I: Haar wavelet illustration

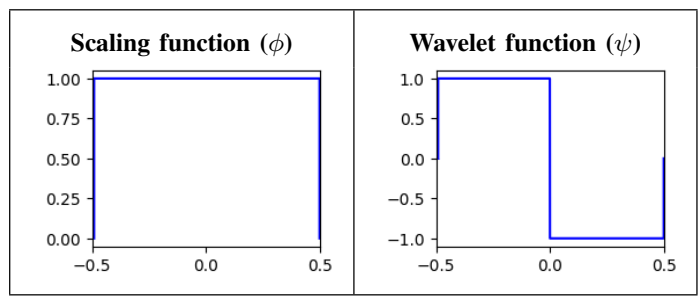

TABLE II: Custom Orthogonal Wavelet (COW) illustration
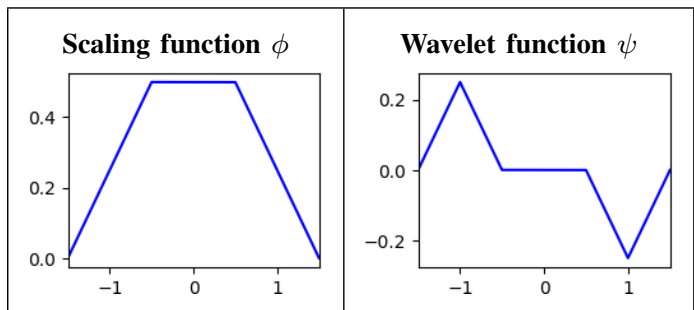

3. Cohen-Daubechies-Fauveau (CDF 9/7) 9/7 wavelet [21], having a great capacity to extract textures, is illustrated in Tab. IV.

4. An Optimized CDF 9/7 9/7 wavelet proposed in [22] and illustrated in Tab. V.

Our goal is to find which wavelet match the most the relevant information in the image, that is, structures salience. Tab. VI shows obtained sharpness maps. We visually estimate that COW seems to match the interesting information, that is, relative to structures' salience. Contrary to CDF 9/7 9/7 wavelet, COW seems to avoids a great part of the image information relative to thermal noise and textural grain.

\section{B. Our IQA method}

In [19], $\mathrm{Vu}$ and Chandler also proposed a wavelet-based algorithm for estimating both the global and local image sharpness (Fast Index SHarpness - FISH). Their IQA comes from the log-energy calculation of a three-level separable Discrete Wavelet Transform (DWT) using CDF 9/7 9/7 wavelet. The choice of this wavelet can be justified by its effectiveness to match textures, and thus to extract a large range of data. The energy evaluation brings information about the overall data values. The sharper an image is, the more its DWT contains high frequencies; and the more there are sparse frequencies, the higher the energy is. Here the sharpest image could be either a well focused image, or an unfocused one, because each contains sparse high frequencies.

We generalize a DWT analysis as follows:

1) 2D DWT of grayscale image using a given wavelet, on $N$ levels. Let $X Y_{n}$ denote the DWT subbands at each level $n$, where $X Y$ is either $L H, H H$ or $H L$ DWT subbands.

2) Given a statistic function $F, F_{X Y_{n}}$ is $F$ computed on each subband $X Y$ at each decomposition level $n$.

3) For each decomposition level $n, F_{n}$ is the pounding sum of each $F_{X Y_{n}}$ :

$$
F_{n}=\alpha F_{L H_{n}}+\beta F_{H L_{n}}+\gamma F_{H H_{n}}
$$

TABLE III: COW filter bank

\begin{tabular}{|c|c|c|c|c|}
\hline $\mathbf{k}$ & Analysis LPF & Analysis HPF & Synthesis LPF & Synthesis HPF \\
\hline 0 & 0.35355339 & -0.35355339 & 0.35355339 & 0.35355339 \\
\hline 1 & 0.35355339 & 0.35355339 & 0.35355339 & -0.35355339 \\
\hline 2 & 0.35355339 & -0.35355339 & 0.35355339 & 0.35355339 \\
\hline 3 & 0.35355339 & 0.35355339 & 0.35355339 & -0.35355339 \\
\hline
\end{tabular}

TABLE IV: CDF 9/7 9/7 wavelet illustration

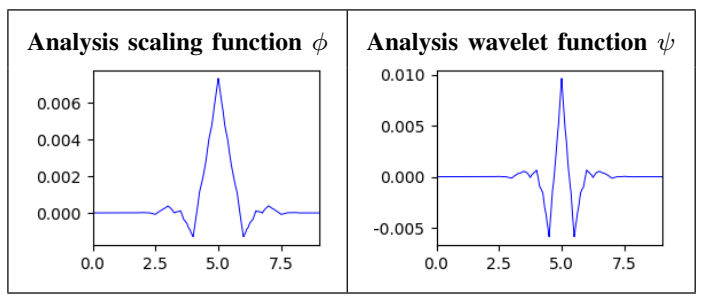


TABLE V: Optimized CDF 9/7 9/7 wavelet illustration

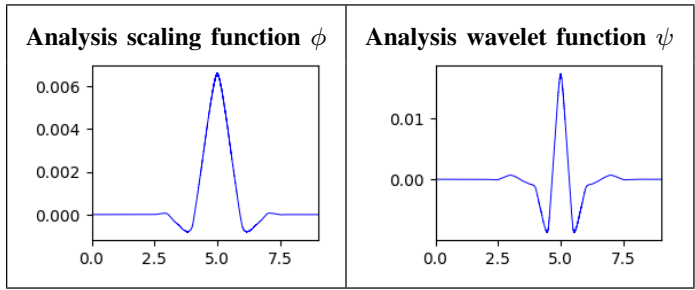

where $\alpha, \beta$ and $\gamma$ are coefficients with $\alpha+\beta+\gamma=1$.

4) The total $F_{\text {tot }}$ of the DWT is the sum of each $F_{n}$ weighted as follows:

$$
F_{\text {tot }}=\sum_{n=1}^{N} 2^{N-n} F_{n}
$$

Our algorithm follows these four steps, with the specifics listed below:

- According to the study in section IV-A, COW is used to match the relevant image information.

- We consider the Standard Deviation (SD) (4), reflecting the image data variability or diversity, since we cannot rely on statistics based on spatial arrangement information, or on its quantity/quality estimation (see section III-B).

$$
S D_{X Y_{n}}=\sqrt{\frac{1}{C} \sum_{c=1}^{C}\left(S_{X Y_{n}}(c)-\mu\right)^{2}}
$$

where $\mu$ is the mean of the considered subband, and $c$ is the coefficient number of this subband from 1 to $C$.

- Only the $H L$ and $L H$ subbands (5) are used: horizontal and vertical components are kept, leaving the diagonal information held by $H H$ (see section III-B).

$$
S D_{n}=\frac{S D_{L H}+S D_{H L}}{2}
$$

- The weighting to obtain $S D_{\text {tot }}$ is reversed compared to $F_{\text {tot }}$ described below:

$$
S D_{\mathrm{tot}}=\sum_{n=1}^{N} 2^{\mathrm{n}} S D_{\mathrm{n}}
$$

For each decomposition level, some information are dropped due to the sub-sampling. Then, in the highest level, the root image information remains.

With this IQA we are then able to rank images by their estimated quality. Our AF method is based on this criterion: the best ranked image in a video corresponds to the focused image, from which the focus position can be estimated (see next section).

\section{EXPERIMENT AND DISCUSSION}

Considering a linear movement given to the optical system toward the OOI, we take a video. The initial position is the farthest position from the OOI, the last is the nearest possible (the physical limits). We make such an acquisition for each magnifying lenses $(2.5 x, 5 x, 20 x$ and $50 x)$, at 80 FPS. From a video, images are extracted so that each image correspond to a lens position, and are evaluated with our proposed quality metric. In this way, the highest-ranked image gives the best matching focus according to our quality criterion.

\section{A. Results}

We firstly compare our algorithm to FISH [19]. Fig. 8 presents the results.

The two approaches have different behaviors. With every magnifying lens, our algorithm effectively catch the SOI focus position (the star in Fig. 8); however, we observe unexpected behaviors around the focus point, which could correspond to optical distortions. The FISH algorithm catch the SOI focus position, but is largely more sensitive to distortions from the optics, the camera and other non-relevant information. This reflects the importance of the textural overcoming the structural information. This sensitivity bias the result, which is inoperable for $2.5 \mathrm{x}$ and $5 \mathrm{x}$ magnifying lenses. These results show that our method is adapted and robust to our specifics.

In order to better describe the image characteristics, we compute other statistics on the same scheme as our algorithm: entropy, Auto-Correlation (AC), skewness and kurtosis. See Fig. 9.

Considering the interpretation of each statistic applied to images, these measures reveal a complex image information. Dis-focused or not, images are saturated: in natural scenes, dis-focused images are considered as blurred. In this case the image data are smoothed and contains little information. In our study the textural part of the data is strong, and light distortions implied by the optical system add unexpected features to the images. Then, even using the DWT with our COW, spatial information is too noisy to be interpreted without preprocessing. The SD measure has a greater abstraction level, enough to remain a reliable quality criterion.

\section{B. Runtime}

As studied in [23] and [24], the computational complexity of DWT using filter banks implementation is $O(n)$. Our algorithm threat the wavelets decomposition linearly per subband. Since only two subbands per level are considered, and for a three-level DWT, the global complexity is around:

$$
O(n)+2 O\left(\frac{n}{2}\right)+2 O\left(\frac{n}{4}\right)+2 O\left(\frac{n}{8}\right) \approx O(n)
$$

We implemented our architecture in Python language, on an 64-bit PC with an Intel Core i5-6200U at $2.30 \mathrm{GHz}$ and $8 \mathrm{~Gb}$ of RAM. The camera resolution is $320 \times 256$ pixels. Additional packages are used for matrix operations (NumPy) and for DWT (PyWavelet) using filter banks.

For a video containing 1500 images, our implementation run in around 3.37 seconds. Then, an image quality is computed in $2.24 \mathrm{~ms}$. In our application, this time is not disturbing: the focus research can be reduced if a previous AF is already done. This solution may considerably reduce video length and so the image stack. 
TABLE VI: Sharpness map in function of magnifying factor and wavelet in use

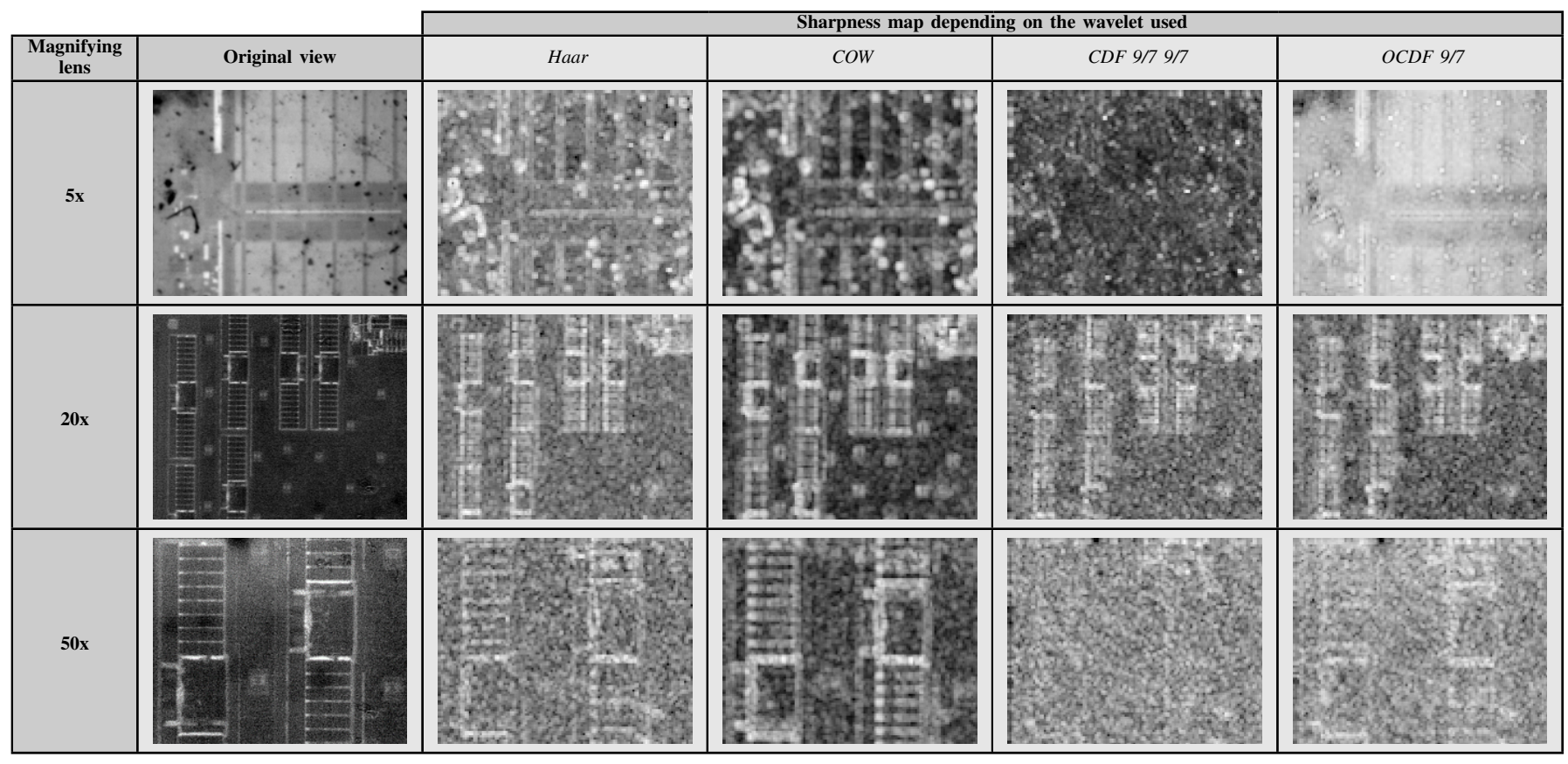

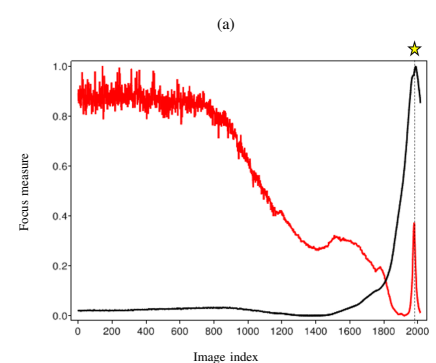

Image index

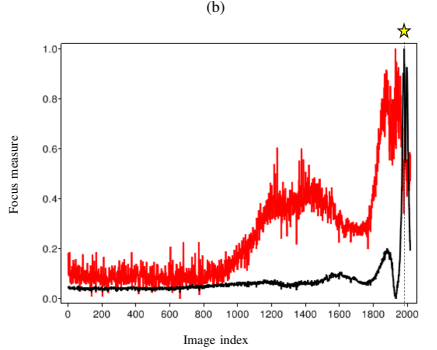

Image index
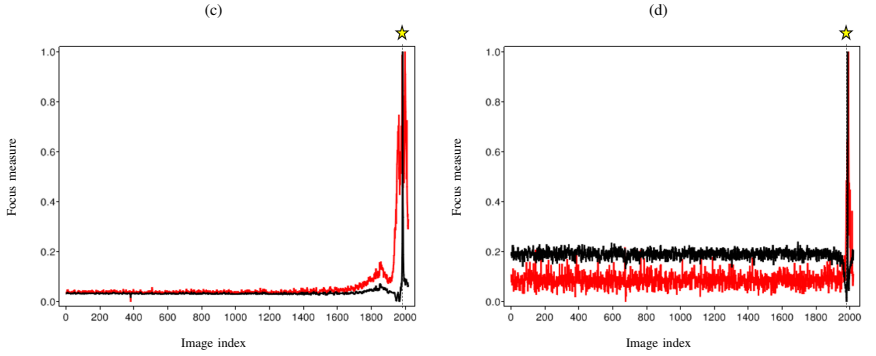

Fig. 8: Graphs of focus measures of images extracted from videos, based on FISH (red) and on ours (black), for different magnifying factors: $2.5 \mathrm{x}(\mathrm{a}), 5 \mathrm{x}(\mathrm{b}), 20 \mathrm{x}(\mathrm{c})$ and $50 \mathrm{x}(\mathrm{d})$; the stars mark the real SOI focus positions.

\section{Evaluation of silicon thickness}

As said in Section I, two adjustments are necessary before a laser shot: the 3D positioning, and the laser power. The laser power setting depends on the silicon thickness on the SOI. A simple way to measure the silicon thickness consists in subtracting the positions of the SOI (1) and the silicon surface (2). The SOI position is available with the proposed algorithm. Changing our AF criterion may permit to focus on (2) instead of (1). For example, in the case of contaminated silicon surface, the sharpness could be a good criterion.

\section{CONCLUSION}

We proposed a robust passive autofocus algorithm. Our quality criterion allows us to efficiently focus on the conductive track surface inside an integrated circuit, independently of the magnifying lens. This proposal responds to a need for accuracy and reproducibility improvement in the context of the security characterization of integrated circuits using laser shots. The proposed algorithm is based on wavelet decomposition analysis, using a wavelet designed for our specifics.
Other approaches may be adopted to study our IR images, as steerable pyramid transforms, or any image-based approach, but they require some preprocessing. Note that data such as the diagonal details of the DWT decomposition stay untapped, and may also be used for IR images denoising. Despite the optical system limitations arising from the uncooled camera, the proposed approach does not need any image enhancement; its time efficiency makes our autofocus method usable for onthe-fly characterizations.

\section{REFERENCES}

[1] Z. Wang, A. C. Bovik, and L. Lu, "Why is image quality assessment so difficult?" in IEEE International Conference on Acoustics Speech and Signal Processing. IEEE, may 2002.

[2] W. S. Streijl R and Hands D, "Mean opinion score (MOS) revisited: methods and applications, limitations and alternatives." Multimedia Systems, 2016.

[3] H. Sheikh and A. Bovik, "Image information and visual quality," IEEE Transactions on Image Processing, vol. 15, no. 2, pp. 430-444, feb 2006.

[4] V. Kamble and K. M. Bhurchandi, "No-reference image quality assessment algorithms: A survey," Optik - International Journal for Light and Electron Optics, vol. 126, no. 11-12, pp. 1090-1097, jun 2015. 

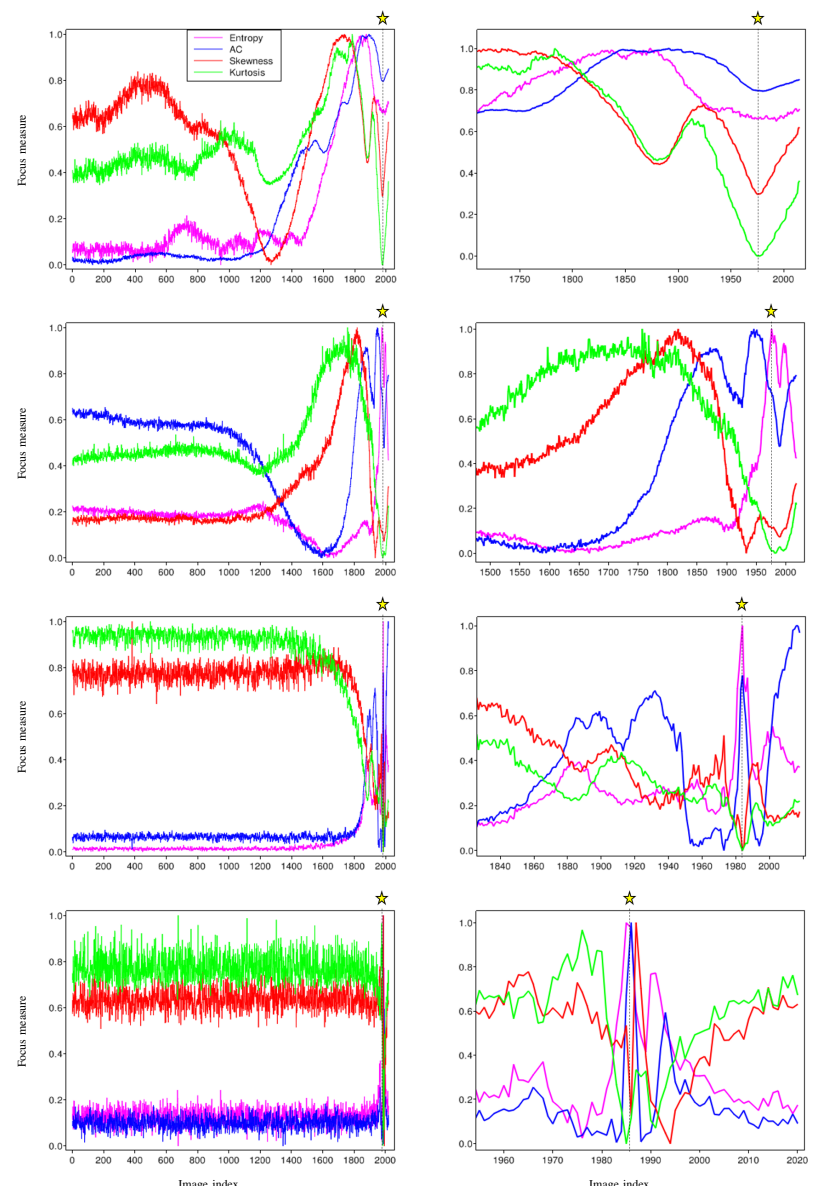

Fig. 9: Graphs of statistics measures of images extracted from videos, following our method, for different magnifying factors: $2.5 \mathrm{x}$ (a), 5x (b), 20x (c) and 50x (d); the AC, kurtosis, entropy, AG and SD are in green, magenta, blue, red and black, respectively. The entire graphs are on the left, whereas zoomed in views around the correct focus index are on the right; the stars mark the real SOI focus positions.

[5] H. Kim, S. Kang, H. Kang, Y. Cho, N. Park, and J. Kim, "Automatic focus control for assembly alignment in a lens module process," in 2009 IEEE International Symposium on Assembly and Manufacturing. IEEE, nov 2009.

[6] V. Kumar and P. Gupta, "Importance of statistical measures in digital image processing," International Journal of emerging technology and advanced engineering, vol. 2, no. 8, pp. 56-62, Aug. 2012.

[7] J. F. Schlag, A. C. Sanderson, C. P. Neuman, and F. C. Wimberly, "Implementation of automatic focusing algorithms for a computer vision system with camera control," CARNEGIE-MELLON UNIV PITTSBURGH PA ROBOTICS INST, Tech. Rep., 1983.

[8] E. Krotkov, "Focusing," International Journal of Computer Vision, vol. 1, no. 3, pp. 223-237, oct 1988.

[9] X. Zhang, H. Wu, and Y. Ma, "A new auto-focus measure based on medium frequency discrete cosine transform filtering and discrete cosine transform," Applied and Computational Harmonic Analysis, vol. 40, no. 2, pp. 430-437, mar 2016.

[10] X. Xu, Y. Wang, J. Tang, X. Zhang, and X. Liu, "Robust automatic focus algorithm for low contrast images using a new contrast measure," Sensors, vol. 11, no. 12, pp. 8281-8294, aug 2011.

[11] J. M. Mateos-Pérez, R. Redondo, R. Nava, J. C. Valdiviezo, G. Cristóbal, B. Escalante-Ramírez, M. J. Ruiz-Serrano, J. Pascau, and M. Desco, "Comparative evaluation of autofocus algorithms for a real-time system for automatic detection of mycobacterium tuberculosis," Cytometry Part A, vol. 81A, no. 3, pp. 213-221, jan 2012.

[12] S. Podlech, "Autofocus by bayes spectral entropy applied to optical microscopy," Microscopy and Microanalysis, vol. 22, no. 01, pp. 199207, jan 2016.

[13] X. Zhang, C. Jia, and K. Xie, "Evaluation of autofocus algorithm for automatic dectection of caenorhabditis elegans lipid droplets," Progress in Biochemistry and Biophysics (PBB), vol. 43, pp. 167-175, 2016, 11.

[14] M. A. Green and M. J. Keevers, "Optical properties of intrinsic silicon at 300 k," Progress in Photovoltaics: Research and Applications, vol. 3, no. 3, pp. 189-192, 1995.

[15] M. A. Green, "Self-consistent optical parameters of intrinsic silicon at $300 \mathrm{k}$ including temperature coefficients," Solar Energy Materials and Solar Cells, vol. 92, no. 11, pp. 1305-1310, 2008.

[16] E. Simoncelli and W. Freeman, "The steerable pyramid: a flexible architecture for multi-scale derivative computation," in Proceedings., International Conference on Image Processing, vol. 3. IEEE Comput. Soc. Press, 1995, pp. $444-447$.

[17] P. Pad, V. Uhlmann, and M. Unser, "VOW: Variance-optimal wavelets for the steerable pyramid," in 2014 IEEE International Conference on Image Processing (ICIP). IEEE, oct 2014, pp. 2973-2977.

[18] P. Hill, A. Achim, M. E. Al-Mualla, and D. Bull, "Contrast sensitivity of the wavelet, dual tree complex wavelet, curvelet, and steerable pyramid transforms," IEEE Transactions on Image Processing, vol. 25, no. 6, pp. 2739-2751, jun 2016.

[19] P. V. Vu and D. M. Chandler, "A fast wavelet-based algorithm for global and local image sharpness estimation," IEEE Signal Processing Letters, vol. 19, no. 7, pp. 423-426, jul 2012.

[20] A. Haar, "Zur theorie der orthogonalen funktionensysteme," Mathematische Annalen, vol. 69, no. 3, pp. 331-371, 1910

[21] A. Cohen, I. Daubechies, and J.-C. Feauveau, "Biorthogonal bases of compactly supported wavelets," Communications on Pure and Applied Mathematics, vol. 45, no. 5, pp. 485-560, jun 1992.

[22] S. Zhang, G. Yang, Z. Cheng, and H. van de Wetering, "A novel 9/7 wavelet filter banks for texture image coding," International Journal of Advanced Research in Artificial Intelligence, vol. 1, no. 6, 2012.

[23] O. Rioul and M. Vetterli, "Wavelets and signal processing," IEEE signal processing magazine, vol. 8, no. 4, pp. 14-38, Oct 1991.

[24] K. K. Shukla and A. K. Tiwari, Efficient algorithms for discrete wavelet transform: with applications to denoising and fuzzy inference systems. Springer Science \& Business Media, 2013.

\section{GLOSSARY}

AC Auto-Correlation. 2, 5

AF Autofocus. 1, 2, 3, 5

AG Average Gradient. 2

CDF 9/7 Cohen-Daubechies-Fauveau 9/7. 4

COW Custom Orthogonal Wavelet. 4, 5, 4

DWT Discrete Wavelet Transform. 1, 4, 5, 6

FR Full-Reference. 2

IC Integrated Circuit. 1, 2, 3

IQA Image Quality Assessment. 1, 2, 3, 4, 5

IR Infrared. 1, 2, 3, 4, 6

NR No-Reference. 2

OOI Object Of Interest. 1, 2, 5

RR Reduced-Reference. 2, 3

SD Standard Deviation. 2, 5

SOI Surface Of Interest. 1, 2, 3, 4, 5

SWIR Short Waves Infrared. 2 